Pure Appl. Chem., Vol. 82, No. 6, pp. 1329-1336, 2010.

doi:10.1351/PAC-CON-10-02-39

(C) 2010 IUPAC, Publication date (Web): 20 April 2010

\title{
Plasma catalysis: A solution for environmental problems*
}

\author{
J. Christopher Whitehead ${ }^{\ddagger}$ \\ School of Chemistry, The University of Manchester, Oxford Road, Manchester M13 \\ 9PL, UK
}

\begin{abstract}
The combination of a nonthermal, atmospheric plasma with a catalyst is investigated as a means of destroying pollutants in waste gas streams. Using the examples of dichloromethane (DCM) and toluene in air streams, it is shown that the destruction of the pollutant can be increased whilst lowering the operating temperature, giving increasing energy efficiency. Unwanted by-products can also be reduced selectively by appropriate choice of catalyst and of the plasma-catalyst configuration. By studying the temperature dependence of plasma catalysis, some ideas can be obtained about the nature of the interaction between plasma and catalyst in the processing.
\end{abstract}

Keywords: atmospheric plasma; catalysis; plasma; pollution; waste gas.

\section{INTRODUCTION}

Plasma methods for the reduction of environmental pollutants have been investigated for nearly 20 years and have been shown to be effective particularly for the removal of small concentrations, typically $<1000 \mathrm{ppm}$, of a wide range of pollutants in waste gas streams. In particular, nonthermal (or lowtemperature) atmospheric-pressure plasma has been demonstrated to possess many desirable features resulting from the operating conditions of the plasma. Working at atmospheric pressure eliminates the need for expensive pumping systems, and low-temperature operation minimizes corrosion compared with competitive remediation technologies such as pyrolysis or thermal catalytic oxidation. Plasma can respond rapidly to changes in the composition of the input gas stream allowing for the adoption of intelligent control strategies and a consequent increase in energy efficiency [1]. A particular advantage is the flexibility of plasma processing and its ability to be combined with other technologies. In this talk, I will focus on the combination of plasma with catalysis and the advantages that this can bring for the remediation of pollutants in waste gas streams.

Plasma-assisted catalysis has the potential to enhance the destruction of the environmental pollutant, vary the selectivity of the processing to minimize unwanted by-products such as $\mathrm{CO}$, organic intermediates, or NOx when processing in air and to reduce the operating temperature of the catalyst which both increases the energy efficiency of the processing and improves the stability of the catalyst by reducing poisoning, coking, and sintering. There have been several previous reviews of plasma catalysis for environmental remediation to which the reader is referred [2-5]. In this presentation, I will discuss the various interactions that plasma can have with a catalyst and illustrate some of these aspects with experimental results from our research group.

\footnotetext{
*Paper based on a presentation at the $19^{\text {th }}$ International Symposium on Plasma Chemistry (ISPC-19), 26-31 July 2009, Bochum, Germany. Other presentations are published in this issue, pp. 1189-1351.

₹E-mail: j.c.whitehead@manchester.ac.uk
} 


\section{INTERACTIONS OF PLASMA WITH A SURFACE}

There are two main configurations in which plasma can be combined with a catalyst. These are illustrated in Fig. 1. In the first, the catalyst is placed in the discharge region (B) and will be referred to as a one-stage configuration. In the second, the catalyst is downstream from the plasma (C) and is called a two-stage arrangement. In a traditional thermal catalysis experiment, molecules are dissociatively adsorbed onto the catalyst with the energy being supplied in the form of heat. In plasma-assisted catalysis, species are activated by the plasma either in the gas phase or on the catalyst surface, with the energy coming from the excitation of the plasma. In a one-stage arrangement, the catalyst is directly in contact with the discharge and the active species are generally short-lived, e.g., excited-state atoms and molecules, reactive radicals, photons, and electrons. In contrast, in the two-stage arrangement, the catalyst is exposed only to species that exit from the plasma region. These will be end-products of the gasphase plasma processing or long-lived intermediates (of which ozone is a common example) and, possibly, vibrationally excited species. In addition, in the one-stage configuration, the role of the plasma may also be to prepare or modify the catalyst surface. Examples of this include the reduction of $\mathrm{NiO}$ to $\mathrm{Ni}$ in a glow discharge in the presence of $\mathrm{Ar}[6]$ and plasma deposition of catalytically active species or plasma modification of catalyst surfaces [7]. In addition, physical properties of the catalyst such as its ability to adsorb species will affect the processing by increasing the lifetime of the adsorbed species in the plasma and hence their reaction times.

(A)

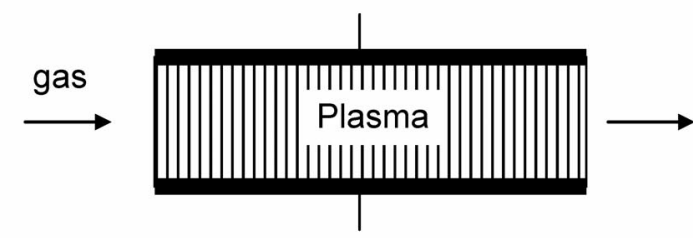

(B)

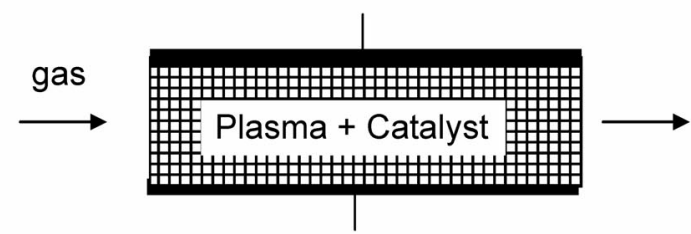

(C)

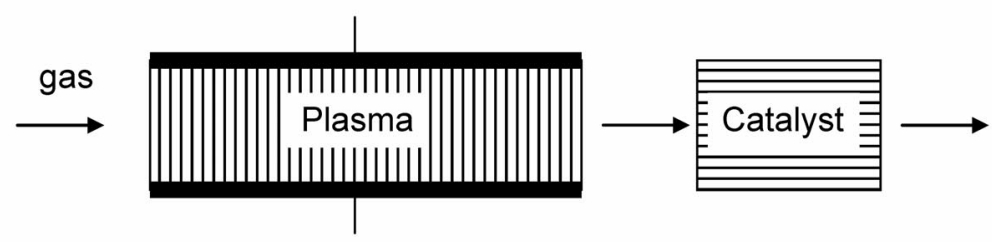

Fig. 1 Schematic diagram of the different plasma-catalyst configurations. Configuration (A) is a plasma-alone configuration without catalyst; configuration (B) places the catalyst directly into the discharge region-a one-stage arrangement; and configuration (C) has the catalyst downstream from the discharge-a two-stage arrangement.

In their excellent review, Chen et al. [5] have distinguished effects in which the catalyst will influence the plasma discharge characteristics from those in which the plasma will affect the catalyst properties. In the former category, the introduction of a packing material (i.e., the catalyst) into the plasma region will change the electrical properties of the plasma (e.g., the electrical field strength), which will change the composition of the reactive species generated or the nature of the discharge from filamentary microdischarges to surface discharges. In the second category, the plasma may generate 
novel or additional reactive species or change the nature of the catalyst. The result of these and other interactions may be to improve the efficiency, selectivity, and stability of the processing. A synergistic effect is sometimes reported where the outcome of employing plasma catalysis exceeds the combined effect of thermal catalysis and plasma operated separately, giving further enhancement in pollutant conversions.

We will now examine various plasma-catalysis experiments that we have studied, which demonstrate some of these aspects.

\section{DESTRUCTION OF CFC-12 USING PLASMA CATALYSIS}

Many methods have been employed to destroy stockpiles of chlorofluorocarbons (CFCs), which are now ozone-depleting species banned under the Montreal Protocol. The strength of the carbon-halogen bond makes them very difficult molecules to destroy. Various techniques, including high-temperature incineration and thermal plasma torches, are used to deal with the different components. Particularly high temperatures $\left(\sim 1250{ }^{\circ} \mathrm{C}\right)$ are needed to ensure that the halogenated components are safely destroyed without the formation of dioxins. We previously studied the destruction of dichlorodifluoromethane, $\mathrm{CCl}_{2} \mathrm{~F}_{2},(\mathrm{CFC}-12)$ in an air stream using only a plasma discharge [8]. By incorporating a range of catalysts into the plasma discharge in a one-stage configuration, we could significantly increase the destruction of the CFC-12 as is shown in Fig. 2 [9]. The plasma reactor is a barium titanate, packed bed to which the different catalysts are added in a powder form between the beads. It can be seen that the catalysts improve the overall destruction in only a modest manner with the exception of $\mathrm{TiO}_{2}$ which increases the percentage CFC-12 destruction from $\sim 12$ to $~ 27 \%$ : an increase of more than a factor of two.

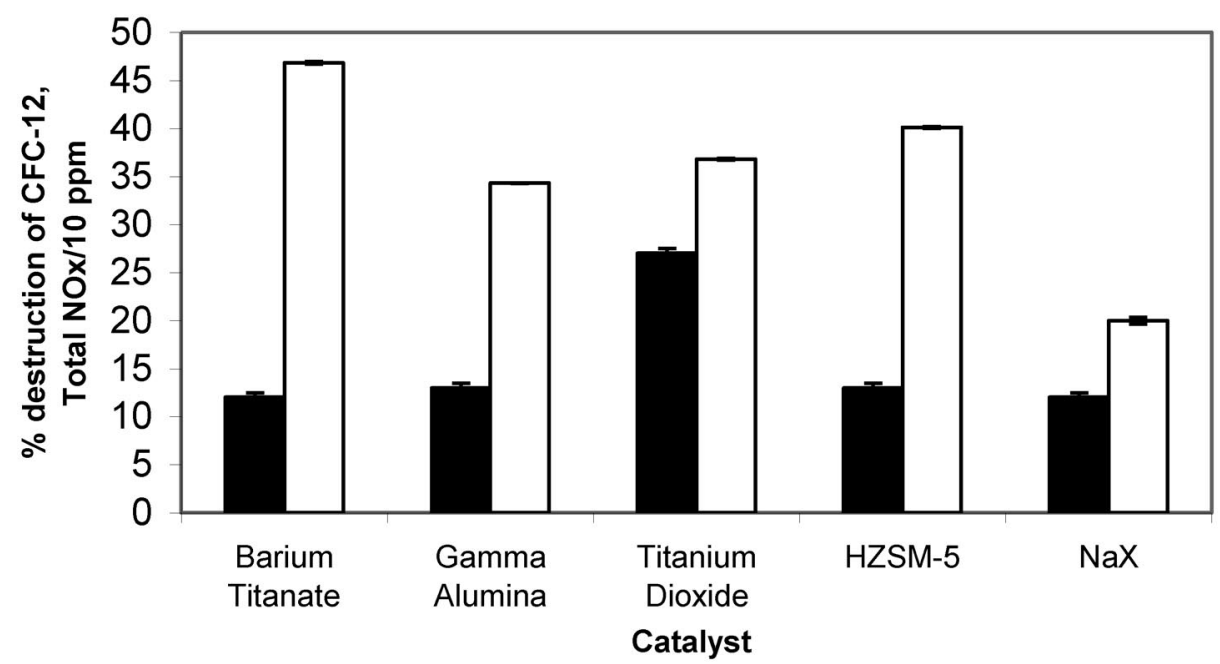

Fig. 2 Chart showing the destruction of $\sim 500$ ppm CFC-12 in an atmospheric-pressure air stream using plasma catalysis with a range of catalysts in a one-stage plasma-catalysis configuration. The plasma is a barium titanate packed bed. The percentage destruction of CFC-12 is designated by solid bars and the NOx concentrations (in $\mathrm{ppm} / 10$ ) by open bars. Barium titanate refers to the plasma reactor without any catalyst packing.

Titanium dioxide is a well-known photocatalyst and has Schottky defects giving n-type semiconductor properties and a bandgap of $3.2 \mathrm{eV}$, which can be activated by UV light of wavelength $<385 \mathrm{~nm}$, which creates electron hole-pair states giving radical cations, $\mathrm{OH}^{-}$from any chemisorbed water and $\mathrm{O}_{2}^{-}$in air-containing systems, with oxidative activity attributed mostly to valence-band holes, 
$\mathrm{O}_{2}, \mathrm{HO}_{2}$, and $\mathrm{H}_{2} \mathrm{O}_{2}$ [10]. Degradation of the CFC-12 occurs through both reductive and oxidative processes on the $\mathrm{TiO}_{2}$ [11]. In the plasma-catalysis system, it is thought [12] that the electrons in the discharge which typically have a mean energy of $\sim 3.5 \mathrm{eV}$ create electron hole-pair states in an analogous manner to UV excitation

$$
\mathrm{TiO}_{2}+e^{-}(>3.2 \mathrm{eV}) \rightarrow \mathrm{h}^{+}+e^{-}
$$

In this case, the plasma discharge is changing the electrical properties of the catalyst.

Inspection of Fig. 2 also shows that the different catalysts affect the production of NOx (NO and $\mathrm{NO}_{2}$ ) to varying extents but in all cases bringing about a reduction compared to the use of the plasma alone. This has been noted for the destruction of other pollutants in air streams and is quite marked in a two-stage configuration, for example, the destruction of dichloromethane (DCM) using a range of zeolite catalysts [13].

\section{TEMPERATURE DEPENDENCE OF PLASMA-ASSISTED CATALYSIS}

It is possible to gain some further insight into the mechanism of plasma-assisted catalysis by studying the temperature dependence of the processing. In these experiments, we again use a dielectric, barium titanate, packed-bed plasma reactor which can be heated up to $400{ }^{\circ} \mathrm{C}$. The catalysts are combined with the plasma whose input power is $1 \mathrm{~W}$, which is determined by integration of the current and voltage waveforms with a typical precision of $\pm 10 \%$ [8].

\section{DICHLOROMETHANE}

DCM is a known hazardous air pollutant and volatile organic compound (VOC), is used extensively as a solvent in a wide range of industrial applications, and has been widely studied by plasma methods in this laboratory and elsewhere. In the heated reactor, we studied [9] the plasma-assisted catalytic processing in three ways and the results are displayed in Fig 3. Firstly, using plasma alone with no catalyst where we see no significant dependence in the destruction with temperature above $\sim 250{ }^{\circ} \mathrm{C}$, giving a destruction of $\sim 20 \%$ (upper panel). A second study was made of the effect of heating on the catalyst alone, i.e., conventional thermal catalysis. It can be seen that there is very little DCM destruction until the temperature rises above $200{ }^{\circ} \mathrm{C}$ when the destruction begins to rise steadily to reach $\sim 80 \%$ at $400{ }^{\circ} \mathrm{C}$, the maximum temperature achievable in the reactor. Finally, we study the effect of temperature on the plasma combined with the catalyst. Whilst the shape of the curve is similar to that measured for thermal catalysis, it starts from a higher baseline ( $10 \%$ destruction) due to the plasma action and is displaced to lower temperatures than thermal catalysis for a given destruction. At $50 \%$ destruction, the lowering is equivalent to a reduction in the operating temperature of $\sim 50{ }^{\circ} \mathrm{C}$. Whilst the plasma energy is only $1 \mathrm{~W}$, the electrical energy supplied to the heater is up to $60 \mathrm{~W}$. For plasma-assisted catalysis, an electrical heating power of $24 \mathrm{~W}$ is required to achieve $50 \%$ destruction of the DCM. For thermal catalysis (without the plasma), $38 \mathrm{~W}$ is required. This shows that an energy saving of $\sim 34 \%$ is achieved using the plasma-catalyst combination; Fig. 3 (lower panel). 

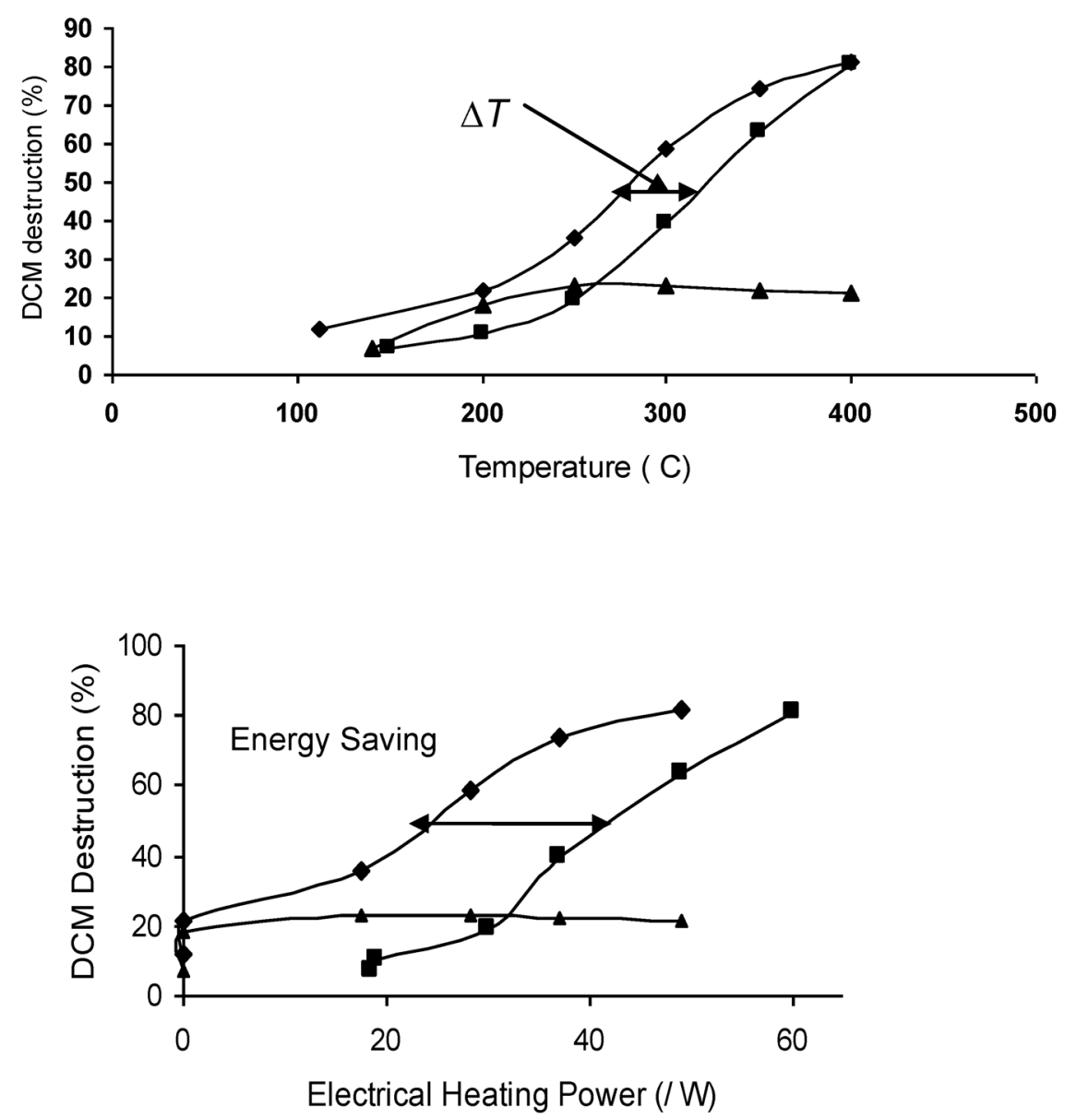

Fig. 3 Percentage destruction of 500 ppm of DCM in atmospheric-pressure air studied as a function of heater temperature (upper panel) and electrical heating power (lower panel) for plasma alone ( $\mathbf{\Lambda})$, plasma catalysis $(\bullet)$ and thermal catalysis ( $\square$ ). The catalyst is $\mathrm{TiO}_{2}$, and the plasma is a barium titanate packed bed.

\section{TOLUENE}

A different type of behavior was observed [14] for the destruction of toluene in atmospheric-pressure air by plasma catalysis using a one-stage, packed-bed plasma system. The results are shown in Fig. 4. Processing in the absence of a catalyst gives low destruction as was seen in the case of DCM, and the use of $\mathrm{TiO}_{2}$ as a catalyst for plasma-assisted catalysis gives similar results for the temperature dependence as did the same catalyst with DCM. However, the use of silver on alumina and titania, $\mathrm{Ag} / \gamma-\mathrm{Al}_{2} \mathrm{O}_{3}$ and $\mathrm{Ag} / \mathrm{TiO}_{2}$, show very different temperature behaviors. There is a large difference in the destruction of toluene between the $\mathrm{TiO}_{2}$ support and the Ag-impregnated $\mathrm{TiO}_{2}$ catalyst. At $257{ }^{\circ} \mathrm{C}$, there is $68 \%$ toluene destruction using the $\mathrm{Ag} / \mathrm{TiO}_{2}$ catalyst, in comparison to only $19 \%$ for the $\mathrm{TiO}_{2}$ support. By $400{ }^{\circ} \mathrm{C}, 100 \%$ conversion is achieved with the plasma- $\mathrm{Ag} / \mathrm{TiO}_{2}$ catalyst combination. A similar but less efficient behavior is found for plasma-catalytic destruction of toluene with the $\mathrm{Ag} / \gamma-\mathrm{Al}_{2} \mathrm{O}_{3}$ catalyst. 


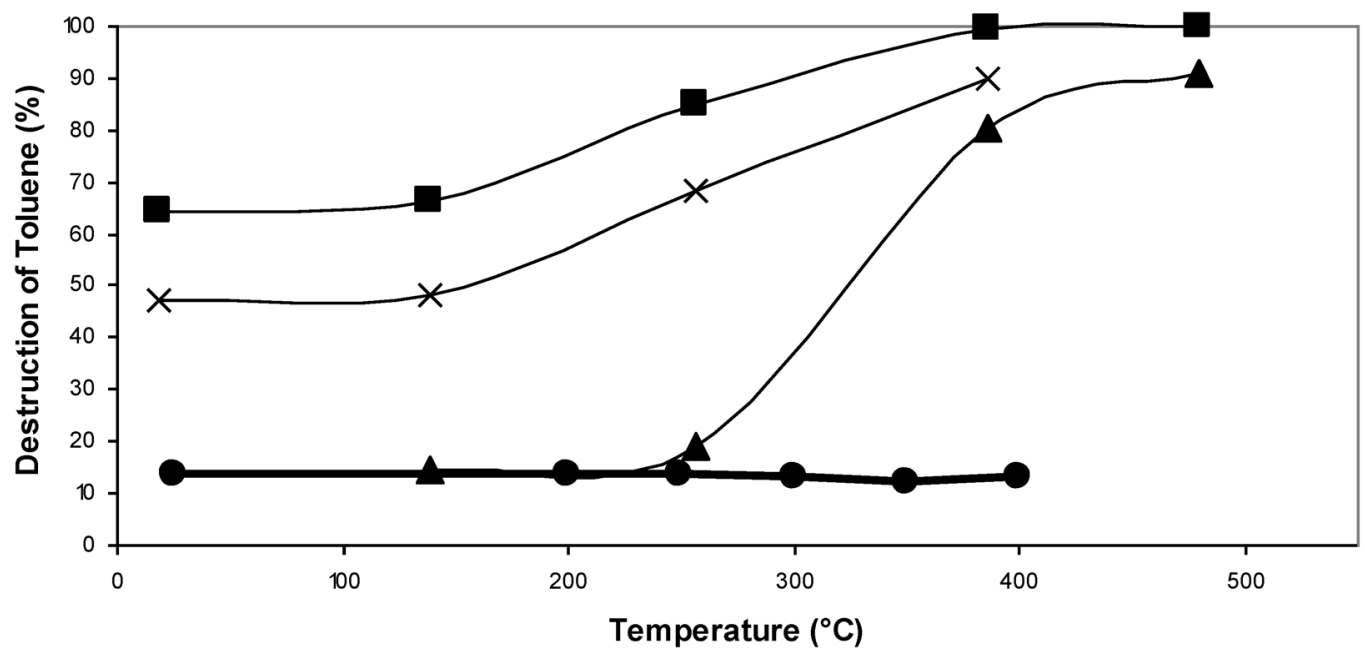

Fig. 4 Comparison of catalyst performance for the plasma-catalytic destruction of $500 \mathrm{ppm}$ of toluene in atmospheric-pressure air at varying temperatures, using $(\boldsymbol{\square}) \mathrm{Ag} /-\mathrm{Al}_{2} \mathrm{O}_{3},(\mathbf{\Delta}) \mathrm{TiO}_{2}$, and $(\times) \mathrm{Ag} / \mathrm{TiO}_{2}$ catalysts and supports, in a one-stage configuration. The thick black line (๑) shows plasma-alone results, with no catalyst. The plasma is a barium titanate packed bed. Adapted from ref. [14].

From this data, we can distinguish two extreme types of behavior exhibited in the temperature dependence of the destruction of toluene by plasma catalysis in a one-stage configuration. The first is highly efficient and only weakly dependent on temperature (e.g., $\mathrm{Ag} / \mathrm{TiO}_{2}$ ) in contrast to the behavior observed in conventional thermal catalysis (e.g., Fig. 3 upper) which shows a rapid rise in the removal of the pollutant above a sharply defined temperature threshold. However, there are also cases where plasma catalysis shows a temperature profile similar to that for thermal catalysis such as the plasmacatalytic destruction of toluene using $\mathrm{TiO}_{2}$ in a one-stage configuration (Fig. 5). It should be noted however, that plasma-activated catalysis is always more efficient than thermal at all temperatures. The existence of these two different generic temperature profiles for plasma-activated catalysis has been taken to indicate that there may be two contributing mechanisms for plasma-activated catalysis; the first being virtually temperature-independent, whereas the second is temperature-dependent with a threshold at a certain temperature in a similar manner to thermal catalysis but generally shifted to lower temperatures. The first type of curve, which is only slightly temperature-dependent, reflects the behavior of the plasma alone where conversions are caused predominantly by gas-phase chemistry. However, it is also possible that in a one-stage configuration, there may be some plasma-induced activation of the catalyst. This could either be by electrons or photons created directly by the discharge (electron-induced processes and production of electrons have only a slight temperature dependence [15]) or by the production of active species such as atomic oxygen or ozone which can then adsorb onto the catalyst surface. For the strongly temperature-dependent curve, thermal activation of the catalyst becomes more important, overtaking the contribution of the plasma-activated processes [16].

The synergistic effect of combining plasma with catalysis is shown in Fig. 5 for the processing of toluene in air by focusing on the data at a low temperature, $25^{\circ} \mathrm{C}$. From left to right, the contributions to the destruction are from plasma alone and from thermal catalysis alone. These are combined to give the resultant effect that one might predict if the two effects were additive $(\sim 20 \%)$. The actual extent of destruction achieved at $25^{\circ} \mathrm{C}$ is shown in the right-hand bar $(\sim 65 \%)$; a synergistic gain of more than a factor of three. However, at higher temperatures there is a much less marked difference between the destruction achieved thermally and by plasma catalysis and this synergy disappears. No synergistic effects 


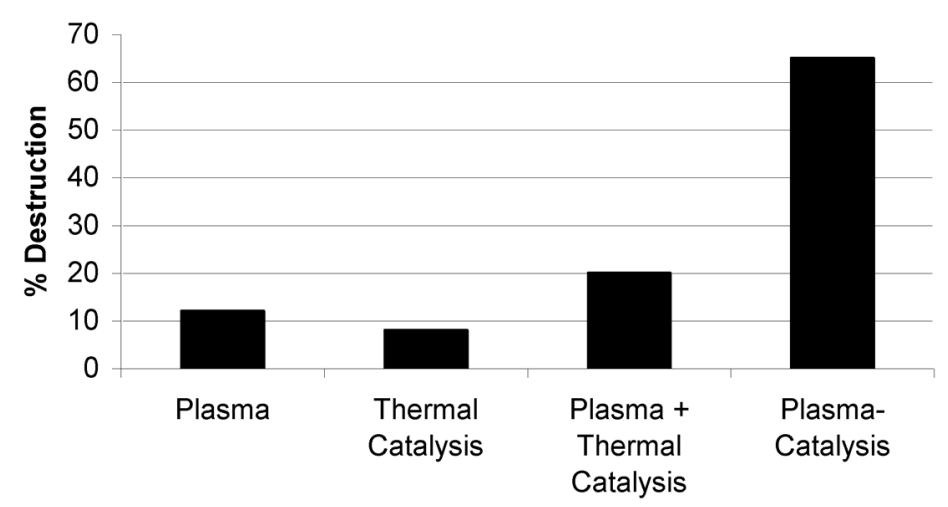

Fig. 5 Demonstration of the "synergy" of plasma catalysis for the destruction of toluene in air detailed in Fig. 4. The operating temperature is $25^{\circ} \mathrm{C}$. The different components to the chart are described in the text.

were observed in our study of the destruction of DCM in air using plasma catalysis. Clearly, the common perception of plasma catalysis universally being a synergistic phenomenon is false.

Another way in which information about the mechanism of plasma-assisted catalysis can be derived is to use the temperature-dependent data to extract kinetic information via an Arrhenius-type analysis. We have performed such an analysis for the destruction of toluene in an atmospheric-pressure air stream using thermal catalysis and plasma-assisted catalysis in both a one- and two-stage system [17]. The catalysts used were $\mathrm{Ag} / \gamma-\mathrm{Al}_{2} \mathrm{O}_{3}$ and $\mathrm{Mn}_{2} \mathrm{O}_{3} / \gamma-\mathrm{Al}_{2} \mathrm{O}_{3}$. The results are given in Table 1 and show how the increase in destruction for the two catalysts that is observed is achieved in very different ways. It was found that there is no difference between the measured activation energy for thermal catalysis and the two-stage (or downstream) plasma-catalysis arrangement. This would suggest that for these systems, there is no activation of the catalysts in the downstream position caused by migration of longlived species produced in the plasma. On the other hand, it was found that plasma could activate catalyst placed inside of the discharge (one-stage configuration). Plasma treatment decreases the activation energy $\left(E_{\mathrm{a}}\right)$ for the $\mathrm{Ag}$-alumina catalyst in a one-stage arrangement but does not increase the number of active centers on the surface of Ag-alumina (as indicated by the $A$ parameter). In the case of $\mathrm{MnO}_{2}$-alumina, the activation mechanism is different: plasma does not change the activation energy and but it does increase its efficiency due to the formation of additional active centers. It is suggested that these active centers could be explained by a change in the oxidation state of Mn ions under plasma action. Alternatively, the interaction of active molecules (ozone, $\mathrm{O}$ atoms, and radicals) with the catalyst surface can also promote electron transfer from the Mn sites.

Table 1 Parameters of the Arrhenius equation, $k=A$ $\exp \left(-E_{\mathrm{a}} / \mathrm{R} T\right), \mathrm{s}^{-1}$ for the decomposition of toluene in atmospheric-pressure air for different plasma-catalysis configurations. Data taken from ref. [17].

\begin{tabular}{clcc}
\hline Catalyst and system & \multicolumn{1}{c}{$\mathrm{A} / \mathrm{s}^{-1}$} & $\begin{array}{c}\text { Activation energy } \\
\left(E_{\mathrm{a}}\right) / \mathrm{kJ} \mathrm{mol}\end{array}$ \\
\hline $\mathrm{Ag}_{2} \mathrm{O} / \mathrm{Al}_{2} \mathrm{O}_{3}$ & Catalyst only & $80.5 \pm 2.5$ & $63.2 \pm 2.5$ \\
2-stage & $84.0 \pm 2.0$ & $63.9 \pm 2.5$ \\
1-stage & $69.5 \pm 10.5$ & $49.0 \pm 8.1$ \\
$\mathrm{MnO}_{2} / \mathrm{Al}_{2} \mathrm{O}_{3}$ Catalyst only & $46.5 \pm 6.0$ & $38.2 \pm 4.9$ \\
2-stage & $43.0 \pm 2.0$ & $34.1 \pm 4.1$ \\
1-stage & $59.1 \pm 6.0$ & $39.8 \pm 7.5$ \\
\hline
\end{tabular}




\section{CONCLUSIONS}

It has been convincingly demonstrated that the combination of plasma with catalysis offers an effective solution to environmental clean-up of pollutants in waste gas streams. From a phenomenological perspective, we have given examples where the hybridisation of the two techniques can bring about improved efficiency by increasing destruction of the pollutant and reducing the operating temperature and energy requirement for the processing. We have also shown that the use of certain catalysts can give increased selectivity in terms of the production or suppression of end-products. However, the detailed understanding of the fundamental mechanism of plasma-assisted catalysis from both a chemical and physical perspective is still very patchy. We need to know more about the nature of the active sites involved in the catalysis using real-time, in situ probing rather than just relying on chemical and surface analysis of the catalyst after processing. We need to understand the balance between homogeneous and heterogeneous chemistry taking place. We also need to understand more about how the catalyst affects the electrical and gaseous dynamics of the discharge. If we can devise experiments that provide answers to these questions, then we may come closer to the goal of being able to design or select catalysts specifically for plasma-assisted catalysis.

\section{ACKNOWLEDGMENTS}

It is a pleasure to acknowledge financial support from the UK Engineering and Physical Sciences Research Council and from members of the Manchester Plasma Chemistry Group whose work is presented here: Tarryn Blackbeard, Vladimir Demidyuk, Alice Harling, Stuart Fischer, Peter Gorry, Sarah Hall, Claire Ricketts, Anna Wallis, and Kui Zang.

\section{REFERENCES}

1. P. A. Gorry, J. C. Whitehead, J. Wu. Plasma Processes Polym. 4, 556 (2007).

2. T. Hammer, T. Kappes, M. Baldauf. Catal. Today 89, 5 (2004).

3. H.-H. Kim, A. Ogata, S. Futamura. In Trends in Catalysis Research, L. B. Bevy (Ed.), pp. 1-50, Nova Science Publishers (2006).

4. J. Van Durme, J. Dewulf, C. Leys, H. Van Langenhove. Appl. Catal., B 78, 324 (2008).

5. H. L. Chen, H. M. Lee, S. H. Chen, M. B. Chang, S. J. Yu, S. N. Li. Environ. Sci. Technol. 43, 2216 (2009).

6. D. Cheng, X. Zhu, Y. Ben, F. He, L. Cui, C. Liu. Catal. Today 115, 205 (2006).

7. C. Liu, G. P. Vissokov, B. W.-L. Jang. Catal. Today 72, 173 (2002).

8. C. L. Ricketts, A. E. Wallis, J. C. Whitehead, K. Zhang. J. Phys. Chem. A 108, 8341 (2004).

9. A. E. Wallis. Plasma-Catalysis for the Removal of Gaseous Pollutants, Ph.D. thesis, University of Manchester, UK (2005).

10. C. Chen, P. Lei, H. Ji, W. Ma, J. Zhao. Environ. Sci. Technol. 38, 329 (2004).

11. P. Calza, C. Minero, E. Pelizzetti. J. Chem. Soc., Faraday Trans. 93, 3765 (1997).

12. A. E. Wallis, J. C. Whitehead, K. Zhang. Catal. Lett. 113, 29 (2007).

13. A. E. Wallis, J. C. Whitehead, K. Zhang. Appl. Catal., B 72, 282 (2007).

14. A. M. Harling, V. Demidyuk, S. J. Fischer, J. C. Whitehead. Appl. Catal., B 82, 180 (2008).

15. A. M. Harling, H. H. Kim, S. Futamura, J. C. Whitehead. J. Phys. Chem. C 111, 5090 (2007).

16. T. Blackbeard, V. Demidyuk, S. L. Hill, J. C. Whitehead. Plasma Chem. Plasma Processing 29, 411 (2009).

17. V. Demidyuk, J. C. Whitehead. Plasma Chem. Plasma Processing 27, 85 (2007). 